\title{
Comparando as idades à primeira relação sexual, à primeira união e ao nascimento do primeiro filho de duas coortes de mulheres brancas e negras em Belo Horizonte: evidências quantitativas
}

\author{
Andréa Branco Simão* \\ Paula Miranda-Ribeiro** \\ André Junqueira Caetano*** \\ Cibele Comini César ${ }^{* \star * *}$
}

\begin{abstract}
Este trabalho apresenta um estudo sobre as idades à primeira relação sexual, ao primeiro casamento e ao nascimento do primeiro filho de mulheres brancas e negras, de 20 a 29 anos e 50 a 59 anos, em Belo Horizonte. Os objetivos centrais são: verificar as mudanças ocorridas nas idades à primeira relação sexual, ao primeiro casamento e ao nascimento do primeiro filho; e analisar se as diferenças constatadas são mais evidentes quando se considera a coorte de nascimento ou a raça/cor das mulheres. Os dados provêm da pesquisa Saúde Reprodutiva, Sexualidade e Raça/Cor (SRSR), realizada pelo Cedeplar em 2002. A metodologia baseia-se em curvas de sobrevivência, obtidas pelo estimador de Kaplan-Meier e comparadas através do teste log-rank.
\end{abstract}

Palavras-chave: Primeira relação sexual. Primeira união. Primeiro filho. Idade. Raça/cor. Belo Horizonte.

\section{Introdução}

O objetivo deste trabalho é analisar as idades à primeira relação sexual, ao primeiro casamento e ao nascimento do primeiro filho de mulheres brancas e negras (autodeclaradas pretas ou pardas), de 20 a 29 anos e 50 a 59 anos, em Belo Horizonte. As coortes de nascimento referem-se, portanto, às mulheres nascidas entre 1973 e 1982 e de 1943 a 1952. O fato de a fecundidade, atualmente, concentrar-se entre as mulheres com menos de 30 anos (especificamente aquelas de 20 a 24 anos) justifica a análise da coorte mais jovem e, para verificar se houve mudanças expressivas nestas idades, ao longo do tempo, julgou-se apropriada a escolha da coorte mais velha, a qual iniciou o período reprodutivo cerca de 20 a 30 anos antes da mais jovem. Adicionalmente, a inclusão do componente racial, que tem sido pouco explorado pelos trabalhos desenvolvidos nesta área, amplia a análise e possibilita um melhor entendimento das desigualdades raciais no país. Por fim, por ter sido o local de uma das mais recentes pesquisas sobre saúde sexual e reprodutiva no Brasil, o município de Belo Horizonte possui dados atuais e representativos, que permitem análises sobre

\footnotetext{
* Pesquisadora da UFMG/Cedeplar. Doutora em Demografia.

** Professora adjunta da UFMG/Cedeplar e Departamento de Demografia. Doutora em Sociologia.

*** Professor da PUC Minas. Pesquisador do Cedeplar/UFMG. Doutor em Sociologia.

**** Professora adjunta do Departamento de Estatística da UFMG/ Cedeplar. Doutora em Demografia.
} 
atividade sexual, casamento e fecundidade de maneira bastante detalhada.

Duas perguntas conduzem este estudo: existem diferenças nas idades à primeira relação sexual, à primeira união e ao nascimento do primeiro filho entre mulheres brancas e negras, de diferentes coortes de nascimento, em Belo Horizonte? Em caso afirmativo, elas são mais evidentes quando se considera a coorte de nascimento ou a raça/cor autodeclarada pelas mulheres?

Para contextualizar a análise, este trabalho procura mostrar a relação dos eventos analisados com as transformações sociais, econômicas, culturais e políticas ocorridas no país, bem como os significados de cada um dos eventos e a forma com que estes se relacionam com a coorte de nascimento, a escolaridade e a raça/cor da mulher. Apresentam-se, ainda, alguns resultados de pesquisas anteriores, que mostram como as idades à primeira relação sexual, ao primeiro casamento e ao nascimento do primeiro filho vêm se comportando no Brasil nas últimas décadas. A explicitação da fonte de dados, das variáveis e da metodologia utilizadas também faz parte deste artigo, assim como os resultados descritivos e aqueles referentes à análise de sobrevivência.

Os resultados mostram que, embora a idade ao primeiro casamento tenha permanecido praticamente inalterada ao longo do período de tempo que separa as duas coortes de mulheres analisadas, as idades à primeira relação sexual e ao nascimento do primeiro filho diminuíram na coorte mais jovem, sendo que a maior redução ocorreu na idade à primeira relação sexual, a qual passou de 21 para 18 anos. No que diz respeito à comparação entre coortes e entre grupos raciais, os resultados sugerem que a distância entre brancas e negras é menor do que aquela entre as mais jovens e as mais velhas.

\section{O comportamento reprodutivo e sexual das mulheres no Brasil: uma breve revisão da literatura}

Ao longo das últimas décadas, as mulheres brasileiras vêm apresentando mudanças notáveis em seu comportamento reprodutivo. Alguns pesquisadores têm apontado as transformações sociais, econômicas, culturais e políticas, ocorridas no país, como responsáveis pelas variações observadas. Estas mudanças, aliadas à difusão de informações, à assimilação de novas idéias e atitudes reprodutivas, a ideais de famílias menores e ao maior acesso a métodos contraceptivos, atuaram no sentido de alterar os níveis e padrões reprodutivos das brasileiras (MERICK e BERQUÓ, 1983; FARIA, 1989; FARIA e POTTER, 1990; GUPTA e LEITE, 2001). Outros pesquisadores sugerem que as variações também podem ocorrer em função de fatores comportamentais, entre os quais estão as idades em que as mulheres iniciam sua vida sexual, se casam, têm seu primeiro filho e o intervalo entre os nascimentos dos filhos (DAVIS e BLAKE, 1956; BONGAARTS e POTTER, 1983). Aliadas a todos estes fatores, existem indicações de que os níveis e padrões de fecundidade variam em função de características sociodemográficas, tais como coorte de nascimento, escolaridade e categoria de raça/cor (MERRICK e BERQUÓ, 1983; BERCOVICH, 1991; FRIAS e CARVALHO, 1994).

Além disso, a literatura sugere que a idade na qual a mulher tem sua primeira relação sexual exerce um efeito importante sobre a seqüência e o tempo de eventos subseqüentes no processo reprodutivo. Por exemplo, a partir do momento em que a mulher inicia sua vida sexual, ela passa, efetivamente, a estar exposta ao risco de engravidar e de ter um filho nascido vivo. Neste contexto, quanto mais jovem uma mulher inicia sua vida sexual, maior é o seu tempo de exposição ao risco de procriar (POPULATION HANDBOOK, 1992).

Ainda quanto à iniciação sexual, alguns estudos sugerem que, para as mulheres de coortes mais velhas, a virgindade deveria ser preservada até o casamento (MIRANDARIBEIRO,1997). Conseqüentemente, a idade de primeira relação sexual, para estas mulheres, não deveria acontecer antes de cerca dos 21 anos, idade em torno da qual se casavam. Para as mulheres das coortes 
mais jovens, entretanto, a virgindade feminina, embora seja apontada como importante, não está conectada ao casamento (MIRANDA-RIBEIRO, 1997; MOORE, 2004).

Quanto ao casamento, aqui entendido como qualquer tipo de união (formal ou informal), existe, em diversas sociedades, um consenso de que ele é o contexto socialmente sancionado para o nascimento dos filhos. Embora a idade ao primeiro casamento não limite, mas apenas sinalize, o início do período de tempo de exposição efetiva ao risco de procriar, estudos mostram que ela desempenha papel fundamental sobre quando se dará a entrada na maternidade e o número de filhos que uma mulher poderá ter até o final de sua vida reprodutiva (HOPES AND REALITIES, 1995).

O nascimento do primeiro filho constitui um fato extremamente relevante para a muIher, pois marca o início de uma nova etapa em sua vida e determina seu comportamento reprodutivo futuro. Alguns autores assinalam que a idade de nascimento do primeiro filho está associada com a idade dos nascimentos subseqüentes. Mulheres que têm o primeiro filho em idades mais jovens apresentam probabilidade mais elevada de ter um segundo nascimento, ou nascimento de ordens superiores, num espaço de tempo mais curto (BUMPASS, RINDFUSS e JANOSIK, 1978).

Em relação à coorte de nascimento, um estudo sobre a fecundidade nas diferentes regiões brasileiras, a partir de 1903, indica que as gerações que iniciaram o período reprodutivo no começo do século $\mathrm{XX}$, até a década de 40, apresentaram maior estabilidade nos seus padrões de fecundidade. Em contrapartida, as gerações a partir de 1948 são compostas por mulheres cujo período reprodutivo iniciou-se na época em que ocorreram transformações importantes na sociedade brasileira, ocasionando reduções expressivas nas taxas de fecundidade do país (FRIAS e CARVALHO, 1994).

Quanto à escolaridade, pode-se considerá-la uma fonte de conhecimento, um veículo de desenvolvimento socioeconômico e um vetor transformador de atitudes. Assim, as mulheres mais escolarizadas tendem a adiar os eventos que marcam o início do período reprodutivo (CASTRO MARTIN e JUAREZ, 1995). No caso da dinâmica da fecundidade no Rio de Janeiro, entre 1991 e 2000, as mulheres mais escolarizadas e que participavam do mercado de trabalho tinham taxas de fecundidade específicas mais baixas que as demais e apresentavam um padrão cuja cúspide estava no grupo entre 25 e 29 anos (CAVENAGHI e ALVES, 2003). No Brasil, estudos mostram que, dos anos $70 \mathrm{em}$ diante, as mudanças no padrão reprodutivo caracterizaram-se, entre outras coisas, pela concentração da fecundidade no grupo de 20 a 24 anos, o que teve papel fundamental na conformação de um padrão reprodutivo tipicamente jovem (SIMÕES e OLIVEIRA, 1998; WONG, 2000).

Já em relação à categoria raça/cor, alguns estudos sugerem que a fecundidade das mulheres pretas, no Brasil, até a primeira metade do século $\mathrm{XX}$, era menor do que a das brancas. A reversão deste quadro aconteceu no final dos anos 60, quando a fecundidade das brancas declinou de forma vertiginosa. Em relação ao padrão etário da fecundidade, segundo raça/cor, estudos empíricos sugerem que as mulheres negras, por iniciarem sua vida sexual mais cedo e terem, em maiores proporções, mais filhos na adolescência do que as brancas, estão sujeitas a apresentarem um padrão de fecundidade mais jovem (BERCOVICH, 1991; PERPÉTUO, 2000).

No que se refere à situação educacional, a população negra apresenta desvantagem em relação à branca, embora o nível educacional de ambas tenha aumentado nas últimas décadas. Esta situação se reflete nos resultados de fecundidade destes estratos populacionais. No caso das mulheres do Rio de Janeiro, entre 1991 e 2000 , constatou-se que as brancas possuíam TFTs mais baixas do que as negras. Porém, observa-se que as mulheres negras com quatro anos e mais de estudo tinham menos filhos do que as brancas com 0 a 3 anos de estudo. Este resultado ressalta o fato de que o grau de escolaridade, mais do que a cor da pele, influencia os níveis de fecundidade (CAVENAGHI e ALVES, 2003). 
No Brasil, pesquisas sobre sexualidade e comportamento reprodutivo, realizadas na década de 90 , têm mostrado que, embora as mulheres das gerações mais jovens estejam se casando praticamente na mesma idade em que se casavam aquelas das gerações mais antigas, elas estão iniciando sua vida sexual e tendo o primeiro filho cada vez mais cedo (BEMFAM, $1992 \mathrm{e}$ 1996). Os resultados da PNDS - Pesquisa Nacional de Demografia e Saúde, de 1996 (BEMFAM, 1996) revelam uma queda de $9,2 \%$ na idade mediana da primeira relação sexual, quando se compra a coorte de 45 a 49 anos com aquela de 25 a 29 anos, a qual passou de 20,7 para 18,8 anos. Esta mesma pesquisa também mostra que a idade ao primeiro casamento permaneceu praticamente inalterada, girando em torno dos 21 anos para diferentes coortes de mulheres. Adicionalmente, a pesquisa sugere que cerca de $50 \%$ das mulheres com menos de 25 anos, na data da entrevista, não haviam tido filhos e que a idade mediana ao nascimento do primeiro filho, para aquelas com mais de 25 anos, ficou em torno de 22,3 anos. Além destes aspectos, os dados da PNDS 1996 revelam que o aumento na escolaridade resultou em idades medianas mais elevadas de entrada na vida sexual, na vida conjugal e na maternidade.

A seguir, são apresentadas a fonte de dados, as variáveis e a metodologia utilizadas neste estudo.

\section{Fonte de dados, variáveis e metodologia}

\section{Fonte de dados}

Este estudo é baseado nas informações coletadas pela pesquisa Saúde Reprodutiva, Sexualidade e Raça/Cor (SRSR), realizada em 2002 pelo Centro de Desenvolvimento e Planejamento Regional (Cedeplar), da Universidade Federal de Minas Gerais (UFMG), englobando os municípios de Belo Horizonte e Recife. Para fins deste estudo, somente a amostra de Belo Horizonte foi utilizada.

A pesquisa coletou informações que possibilitam a caracterização e a avaliação da situação e da qualidade da saúde reprodutiva da população feminina dos municípios de Belo Horizonte e Recife. Estes dados foram captados de forma a permitir comparações por raça/cor, idade, situação socioeconômica e município de residência, bem como a verificação da existência de diferenças estatisticamente significantes.

Utilizou-se amostragem estratificada em três estágios para a coleta de dados da pesquisa SRSR. No primeiro estágio, o cadastro de setores do IBGE foi usado para estratificar e selecionar 70 setores em cada um dos municípios. Estes setores foram selecionados por meio de amostragem sistemática, com probabilidade proporcional ao tamanho, sendo usado como medida de tamanho o total de domicílios particulares encontrado em cada setor por ocasião do Censo Demográfico de 2000. Em cada um destes setores foi selecionada uma amostra de 22 domicílios, que foram visitados pelas entrevistadoras para cadastramento dos moradores e identificação da população elegível. Nos domicílios com mais de uma moradora elegível, foi sorteada uma mulher para participar da pesquisa (MIRANDA-RIBEIRO e CAETANO, 2003).

No total, foram realizadas 1.301 entrevistas completas com mulheres entre 15 e 59 anos, em Belo Horizonte, as quais são representativas da população feminina do município, neste intervalo etário. Destas mulheres, 361 possuíam entre 20 e 29 anos e 173 tinham entre 50 e 59 anos. Juntas, estas duas coortes somam 534 mulheres. Excluindo-se, porém, aquelas que se autodeclararam amarelas e indígenas, que não fazem parte da população de interesse para o estudo (aproximadamente 5\%), estas duas coortes somam 521 mulheres autodeclaradas brancas, pretas ou pardas, as quais são o alvo deste estudo.

\section{Variáveis utilizadas}

Foram selecionadas variáveis de diferentes seções do questionário e algumas foram recodificadas. Embora exista, no banco de dados, a variável "idade em anos completos", a idade da entrevistada foi gerada a partir das informações de mês e 
ano de nascimento. Este procedimento visou evitar a preferência por dígitos, que pode ocorrer nas declarações de idade relativas a anos completos. Com base na nova variável de idade, foi construída a variável "coorte". No caso desta investigação, existem duas coortes de interesse, a de 20 a 29 anos e a de 50 a 59 anos, denominadas "coorte 1" e "coorte 2", respectivamente.

As idades em que as mulheres experimentaram os eventos investigados também foram construídas a partir das informações disponíveis para mês e ano. As idades, em anos completos foram consideradas somente nos casos em que as mulheres não sabiam informar as datas de ocorrência dos eventos.

A classificação de raça/cor foi feita a partir da resposta dada pelas próprias entrevistadas à pergunta que segue os moldes do IBGE, cujas alternativas de resposta são: 1) branca; 2) preta; 3) parda; 4) amarela e 5) indígena. Nesta análise, as mulheres que se autodeclararam pretas e pardas foram agrupadas na categoria "negra". Com isso, a variável raça/cor, na análise, é composta por duas categorias: 1) brancas e 2) negras (pretas e pardas).

A decisão de agrupar as pretas e as pardas em uma única categoria tem duas razões. Primeiro, porque, como constataram Carvalho, Wood e Andrade (2003), existe uma mobilidade na autodeclaração de cor, sendo que este processo é mais visível entre pardos e pretos. Segundo, porque, como Telles (2003) apontou, há uma probabilidade maior de que entrevistados e entrevistadores concordem mais sobre quem é branco do que sobre quem é preto ou pardo.

\section{Metodologia}

O primeiro passo na análise dos dados é realizar uma caracterização das mulheres de 20 a 29 anos e de 50 a 59 anos. As distribuições segundo coorte de nascimento, escolaridade, raça/cor e situação conjugal são descritas, enquanto aquelas referentes às mulheres que tiveram filhos antes ou depois da primeira união são analisadas. Quando existem freqüências esperadas menores do que cinco, estas distribuições são comparadas por meio do teste exato de Fisher, caso contrário, o teste qui-quadrado de Pearson é utilizado.

O segundo passo é comparar as idades à primeira relação sexual, à primeira união e ao nascimento do primeiro filho, referentes às duas coortes analisadas e às mulheres brancas e negras. Para tanto, as histórias das mulheres da coorte mais velha são reconstruídas para quando elas tinham entre 20 e 29 anos. Isto só é possível porque o banco de dados possui, além da história de nascimentos, perguntas sobre as datas e idades, em anos completos, de primeira relação sexual e primeiro casamento. Esta é uma característica extremamente importante deste banco de dados, pois permite que se conheçam as datas exatas de todos os eventos de interesse.

É importante lembrar, entretanto, que os dados não se referem somente às mulheres com histórias de vida sexual, conjugal e reprodutiva completas. Isto significa que, na data da entrevista, existiam mulheres nas coortes de interesse que ainda não haviam experimentado os eventos analisados neste estudo. Este fato não possibilita conhecer a freqüência exata associada a cada intervalo, ou seja, os dados são censurados. A presença de censura é um problema para as técnicas convencionais de análise descritiva. Para enfrentar esta dificuldade, utiliza-se o método de Kaplan-Meier para estimar funções de sobrevivência, a partir das quais são obtidas as idades medianas de ocorrência dos eventos. Modelos de sobrevivência são adequados para análises em que o tempo de exposição ao risco de experimentar um evento não é o mesmo para todos os indivíduos (ALLISON, 1995). Para realizar esta análise, foi utilizado o pacote estatístico SPSS versão 10.0.

Kaplan-Meier (KM) é um estimador nãoparamétrico, utilizado para estimar funções de sobrevivência, $S(t)$, sendo $t$ a idade de ocorrência do evento de interesse. É importante esclarecer que a função de sobrevivência é a probabilidade de que o tempo de um evento seja maior do que $t$, sendo $t$ qualquer número não negativo (ALLISON, 
1995). No caso deste estudo, a menor idade registrada na pesquisa SRSR, para cada um dos eventos, foi assumida como o tempo de início da observação. Para primeira relação sexual, a menor idade relatada foi de 9 anos; para o primeiro casamento, de 11 anos; e para o nascimento do primeiro filho, de 13 anos. O estimador Kaplan-Meier é definido da seguinte maneira:

$$
\hat{S}(t)=\prod_{j: t ; t t}\left[1-\frac{d_{j}}{n_{j}}\right]
$$

sendo:

$t$ a idade de ocorrência dos eventos;

$n_{j}$ o número de indivíduos sob o risco de experimentar o evento imediatamente antes da idade $t_{j}$;

$d_{j}$ o número de eventos que aconteceram na idade $t_{\mathrm{j}}$.

Para verificar se as diferenças nas distribuições de sobrevivência e, conseqüentemente, nas idades de ocorrência dos eventos são significativas, é utilizado o teste log-rank. Este teste é uma das alternativas estatísticas disponíveis para testar a hipótese nula de que as funções de sobrevivência são iguais entre as populações estudadas (ALLISON, 1995). A expressão estatística do teste log-rank é a seguinte:

$$
\sum_{j=1}^{r}\left(d_{1 j}-e_{1 j}\right)
$$

sendo:

$r$ o número de momentos distintos em que ocorreu o evento;

$d_{1 j}$ o número de eventos que aconteceram no grupo 1 , no tempo j;

$\mathrm{e}_{1 \mathrm{j}} \mathrm{O}$ número esperado de eventos no grupo 1 , no tempo j, sob a hipótese de não haver diferença entre os grupos. Neste caso, o número de eventos esperados é dado por $\mathrm{n}_{1 \mathrm{j}} \mathrm{d}_{\mathrm{i}} / \mathrm{n}_{\mathrm{i}}$, sendo $n_{j}$ o número total de casos sob risco de um evento antes do tempo $j, n_{1 j}$ o número sob risco antes do tempo $j$ no grupo 1 e $d_{j}$ o total de eventos no tempo $j$ nos grupos analisados.

Para comparar as curvas, apresentamse, a seguir, os resultados do teste log-rank. Embora sejam analisadas todas as comparações realizadas, somente os gráficos que apresentam diferenças significativas são exibidos.

\section{Quem são as brancas e negras de 20 a 29 anos e de 50 a 59 anos em Belo Horizonte, em 2002?}

A análise aqui desenvolvida se concentra nas idades que ocorreram a primeira relação sexual, a primeira união e o nascimento do primeiro filho de mulheres brancas e negras, entre 20 e 29 anos (coorte 1) e de 50 a 59 anos (coorte 2), residentes em Belo Horizonte em 2002. O estudo tem por base 521 mulheres ( $40 \%$ do total da amostra de Belo Horizonte), das quais 355 (68\%) tinham entre 20 e 29 anos e 166 (32\%) estavam no grupo etário 50-59 anos.

$\mathrm{Na}$ coorte 1, 158 mulheres (45\%) se autodeclararam brancas e 197 (55\%) negras, enquanto na coorte 2 esses números corresponderam a $86(52 \%)$ e 80 (48\%), respectivamente.

O fato de mais de $50 \%$ das mulheres mais jovens terem se autodeclarado negras não causa surpresa, uma vez que, no Brasil, existe uma certa mobilidade na classificação de cor, a qual pode ser afetada por diversos fatores, sendo um deles a idade (CARVALHO, WOOD e ANDRADE, 2003). Além disso, dada a intensidade da miscigenação entre a população brasileira, é de se esperar que, nas coortes mais jovens, haja mais pessoas autodeclaradas pardas, o que aumentaria a proporção de negros. A Tabela 1 apresenta uma síntese de alguns dos resultados obtidos para estas mulheres.

Em relação à escolaridade, os dados revelam diferenças segundo raça/cor e coorte de nascimento. Na coorte 1 (20 a 29 anos), por exemplo, enquanto $11,7 \%$ das mulheres negras informaram ter de 1 a 4 anos de estudo, apenas $3,8 \%$ das brancas relataram o mesmo. A diferença se inverte para aquelas com 12 anos e mais de estudo, com $27,8 \%$ das brancas presentes na categoria, contra $14,7 \%$ das negras. O teste exato de Fisher mostra que estas diferenças são significativas (valor- $p=0,004$ ). Estes diferenciais também são observados na coorte 2, indicando que, em ambas as coortes, as mulheres brancas apresentam maior escolaridade, apesar da melhora nos níveis educacionais das negras. Assim 
TABELA 1

Distribuição das mulheres de 20 a 29 anos (coorte 1) e de 50 a 59 anos (coorte 2), por raça/cor, segundo características selecionadas

Belo Horizonte - 2002

\begin{tabular}{|c|c|c|c|c|}
\hline \multirow{3}{*}{$\begin{array}{l}\text { Características } \\
\text { selecionadas }\end{array}$} & & & \multicolumn{2}{|c|}{ Em porcentagem } \\
\hline & \multicolumn{2}{|c|}{ Coorte 1} & \multicolumn{2}{|c|}{ Coorte 2} \\
\hline & Brancas & Negras & Brancas & Negras \\
\hline Anos de estudo & 100,0 & 100,0 & 100,0 & 100,0 \\
\hline 0 & 0,6 & 1,0 & 7,0 & 8,8 \\
\hline 1 a 4 anos & 3,8 & 11,7 & 22,1 & 48,8 \\
\hline 5 a 8 anos & 25,9 & 28,9 & 16,3 & 13,8 \\
\hline 9 a 11 anos & 41,8 & 43,7 & 26,7 & 21,3 \\
\hline 12 e mais & 27,8 & 14,7 & 27,9 & 7,5 \\
\hline Situação conjugal & 100,0 & 100,0 & 100,0 & 100,0 \\
\hline Nunca unida & 53,2 & 57,4 & 7,0 & 12,5 \\
\hline Casada/unida & 33,5 & 37,0 & 69,8 & 60,0 \\
\hline Separada/divorciada & 13,3 & 5,1 & 13,9 & 13,8 \\
\hline Viúva & 0,0 & 0,5 & 9,3 & 13,8 \\
\hline Nascimento do $1^{\circ}$ filho & 100,0 & 100,0 & 100,0 & 100,0 \\
\hline Antes da $1^{\mathrm{a}}$ união/mãe solteira & 6,3 & 6,6 & 3,5 & 5,0 \\
\hline Depois da $1^{\mathrm{a}}$ união & 32,3 & 28,9 & 86,0 & 76,3 \\
\hline Nunca teve filho & 61,4 & 62,9 & 10,5 & 18,8 \\
\hline Não informou & 0,0 & 1,5 & 0,0 & 0,0 \\
\hline $\mathrm{N}^{\circ s}$ absolutos & 158 & 197 & 86 & 80 \\
\hline
\end{tabular}

Fonte: Pesquisa SRSR, Cedeplar, Belo Horizonte, 2002.

como no caso anterior, a diferença por raça/ cor também é significativa (valor- $p=0,001$ ).

Quanto à situação conjugal, os dados da pesquisa SRSR revelam que a proporção de mulheres casadas ou unidas, na coorte mais jovem (coorte 1), é superior para as negras (37\%) em comparação com as brancas (34\%). Esta situação se inverte na coorte 2 , com $70 \%$ de brancas e $60 \%$ de negras casadas ou unidas. Em ambos os casos, os resultados não são estatisticamente significantes (valor- $p=0,0505$ e 0,197 , respectivamente).

$\mathrm{Na}$ coorte 1, chama a atenção a diferença observada entre os porcentuais de separadas/divorciadas, quando se comparam os dois grupos de raça/cor. Entre as brancas, $13 \%$ informaram ser divorciadas ou separadas, ao passo que, entre as negras, apenas $5 \%$ declararam estar na mesma situação. Ao nível de significância de $5 \%$, a diferença entre brancas e negras é significativa (valor- $p=0,043$ ).

Outro aspecto interessante revelado pelos dados da pesquisa SRSR refere-se à distribuição de mulheres que tiveram o nascimento do primeiro filho antes ou depois da primeira união. Em Belo Horizonte, os resultados indicam que o nascimento do primeiro filho geralmente acontece dentro de uma união. Na coorte 1 , cerca de $80 \%$ das mulheres tiveram o primeiro filho após terem se unido. Foi possível, entretanto, observar uma variação segundo raça/cor, sendo que as brancas apresentaram proporção mais elevada de nascimentos depois da união do que as negras $(84 \%$ e $78 \%$, respectivamente). Na coorte 2 , cerca de $95 \%$ das mulheres tiveram o primeiro filho depois de casadas. O teste exato de Fisher indica que, tanto no caso da coorte 1 como no da coorte 2, a diferença não é 
estatisticamente significativa (valor- $p=0,518$ e 0,248 , respectivamente). A maior proporção de mulheres com nascimentos préunião, na coorte mais jovem, sugere que houve uma alteração na forma de entrada na maternidade.

A fim de estudar a idade ao nascimento do primeiro filho de mulheres de duas gerações distintas, a parturição das mesmas é verificada em dois pontos: até os 19 anos e até os 24 anos. Como esta análise envolve histórias de nascimentos incompletas, nesta etapa, as seguintes estratégias são utilizadas:

- para comparar a parturição que as duas coortes apresentaram até os 19 anos, as 521 mulheres da amostra selecionada são incluídas na análise;

- para comparar a parturição até os 24 anos, somente as mulheres com mais de 24 anos são incluídas, perfazendo, assim, um total de 319 mulheres.
A Tabela 2 apresenta os resultados das parturições até os 19 anos ${ }^{1}$ e até os 24 anos, para as coortes estudadas.

Verifica-se que, na coorte $1,77 \%$ das brancas e $75 \%$ das negras permaneceram na parturição de ordem zero, ou seja, não tiveram nenhum filho até os 19 anos. Entre aquelas que registraram uma parturição até esta idade, a proporção é ligeiramente mais elevada para as negras (21\%) em comparação às brancas (18\%). Estes resultados são compatíveis com as observações realizadas por Bercovich (1991) e Perpétuo (2000), as quais sugerem que, por apresentarem maiores proporções de filhos na adolescência, as negras estão sujeitas a uma fecundidade mais rejuvenescida do que as brancas.

$\mathrm{Na}$ coorte 2, cerca de $85 \%$ das brancas e das negras tiveram parturição de ordem zero até os 19 anos e em torno de 10\% atingiram a parturição de ordem um até esta mesma idade.

TABELA 2

Distribuição das mulheres de 20 a 29 anos (coorte 1) e de 50 a 59 anos (coorte 2), por raça/cor, segundo número de parturições

Belo Horizonte - 2002

Em porcentagem

\begin{tabular}{lccccc}
\hline \multirow{2}{*}{$\begin{array}{l}\text { Número de } \\
\text { parturições }\end{array}$} & \multicolumn{2}{c}{ Cooorte 1 } & & \multicolumn{2}{c}{ Coorte 2 } \\
\cline { 2 - 3 } \cline { 5 - 6 } & Branca & Negra & & Branca & Negra \\
\hline Parturição até 19 anos & $\mathbf{1 0 0 , 0}$ & $\mathbf{1 0 0 , 0}$ & & $\mathbf{1 0 0 , 0}$ & $\mathbf{1 0 0 , 0}$ \\
0 & 76,6 & 74,5 & & 83,9 & 85,0 \\
1 & 17,7 & 20,9 & & 9,2 & 10,0 \\
2 & 5,1 & 4,1 & & 6,9 & 5,0 \\
3 & 0,6 & 0,5 & & 0,0 & 0,0 \\
4 & 0,0 & 0,0 & & 0,0 & 0,0 \\
$\mathbf{N}^{\text {os }}$ absolutos & $\mathbf{1 5 8}$ & $\mathbf{1 9 6}$ & & $\mathbf{8 7}$ & $\mathbf{8 0}$ \\
\hline Parturição até 24 anos & $\mathbf{1 0 0 , 0}$ & $\mathbf{1 0 0 , 0}$ & & $\mathbf{1 0 0 , 0}$ & $\mathbf{1 0 0 , 0}$ \\
0 & 56,6 & 49,4 & & 41,0 & 57,0 \\
1 & 26,3 & 28,6 & & 26,0 & 10,1 \\
2 & 14,5 & 11,7 & & 21,0 & 20,3 \\
3 & 2,6 & 9,1 & 7,0 & $\mathbf{8 , 9}$ \\
4 & 0,0 & 1,2 & 5,0 & 3,7 \\
$\mathbf{N}^{\text {os }}$ absolutos & $\mathbf{7 6}$ & $\mathbf{7 7}$ & $\mathbf{8 6}$ & $\mathbf{8 0}$ \\
\hline
\end{tabular}

Fonte: Pesquisa SRSR, Cedeplar, Belo Horizonte, 2002.

${ }^{1}$ Nenhuma das comparações descritas para a parturição até os 19 anos apresenta significância estatística. 
A comparação entre as coortes de muIheres de mesmo grupo de raça/cor revela que a proporção de mulheres brancas da coorte 1 que atingiram a parturição de ordem um até os 19 anos é bem superior à da coorte 2. Entre as brancas mais jovens, $18 \%$ chegaram à parturição de ordem um até os 19 anos, enquanto para as brancas mais velhas somente $9 \%$ tiveram um filho até esta idade. Esta situação também se repete entre as negras, para as quais chama a atenção o fato de a parturição de ordem um ser duas vezes maior para as mais jovens, em relação às mais velhas $-21 \%$ e $10 \%$, respectivamente. Isto indica que as mais jovens estão tendo filho mais cedo - cerca de 1/4 delas já haviam tido pelo menos um filho até os 19 anos, contra cerca de menos de $15 \%$ na coorte mais velha.

Os resultados relativos à parturição atingida até os 24 anos indicam que, na coorte 1, a maior proporção de mulheres com parturição de ordem zero até os 24 anos é verificado para as brancas $(57 \%$, contra $50 \%$ entre as negras). Já o porcentual de negras da coorte 1 que chegaram até os 24 anos com parturição de ordem um é mais elevado do que o de brancas - $29 \%$ e $26 \%$, respectivamente. Estas comparações não são significativas (valor- $p=0,361$ ).

Para a coorte 2, um aspecto interessante, revelado pelos resultados relativos às parturições atingidas pelas mulheres brancas e negras até os 24 anos, refere-se ao fato de que, na parturição de ordem um, encontra-se o dobro de mulheres brancas do que de negras $(26 \%$ e $10 \%$, respectivamente). Esta comparação não é significativa (valor- $p=0,088$ ).

Quando se consideram as brancas das duas coortes, observa-se que a proporção de jovens, ou seja, de mulheres da coorte 1 que chegaram à parturição de ordem um até os 24 anos é muito similar à da coorte 2 , ficando em torno dos $26 \%$. Neste caso, o teste exato de Fisher indica que a comparação não é estatisticamente significativa (valor- $p=0,105)$. Já para as negras, o porcentual de jovens com parturição de ordem um até os 24 anos (29\%) é quase três vezes maior do que o verificado entre as mulheres da geração mais velha (10\%). Ao nível de significância de $5 \%$ a comparação entre negras mais jovens e mais velhas é significativa (valor- $p=0,030$ ). Tendo em vista que a variável parturição refere-se a filhos nascidos vivos, uma das possíveis explicações para este resultado pode estar na melhora das condições de saúde das mulheres negras mais jovens, em relação às mais velhas, em particular da saúde reprodutiva (OLIVEIRA, 1999). Adicionalmente, vale ressaltar que, na coorte 2, a parturição de ordem zero é mais alta entre as negras. Este resultado pode estar associado com a proporção de negras que nunca se casaram, ${ }^{2}$ ou com as maiores dificuldades no mercado de casamento enfrentadas pelas negras da coorte mais velha.

As curvas de sobrevivência e seus respectivos testes de significância (logrank), assim como as idades medianas relativas ao início da vida sexual, ao primeiro casamento e ao nascimento do primeiro filho, são apresentadas a seguir.

\section{As idades de interesse}

\section{Idade à primeira relação sexual}

As curvas de sobrevivência apresentadas no Gráfico 1 permitem comparar a idade de início da vida sexual das mulheres da coorte 1 com as da coorte 2 .

As curvas mostram que as mulheres mais jovens iniciaram sua vida sexual mais cedo do que as mulheres da coorte mais velha. O teste log-rank mostra que esta diferença é estatisticamente significante (valor- $p<0,001)$. Adicionalmente, a comparação das idades medianas indica uma diferença de cerca de três anos na idade de início da vida sexual de uma coorte para a outra (18 anos para as mais jovens e 21 anos para as mais velhas).

\footnotetext{
${ }^{2}$ Para maiores detalhes, ver Simão (2005).
} 
GRÁFICO 1

Curvas de sobrevivência da idade à primeira relação sexual, segundo coortes de idade Belo Horizonte - 2002

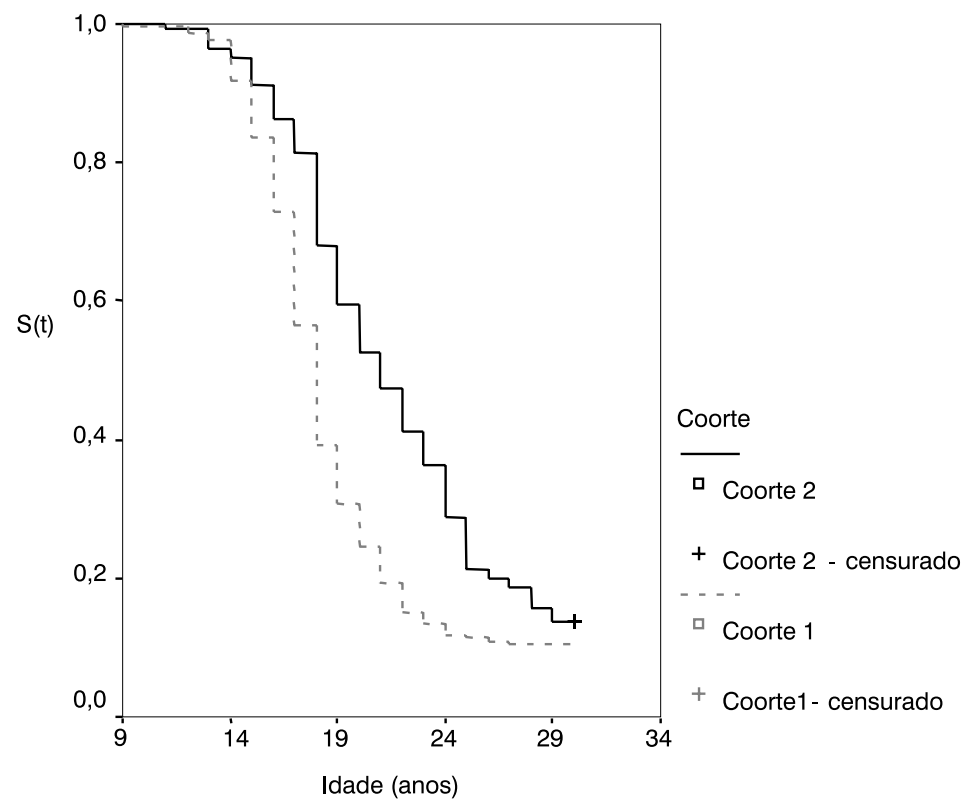

Fonte: Pesquisa SRSR, Cedeplar, Belo Horizonte, 2002.

GRÁFICO 2

Curvas de sobrevivência da idade à primeira relação sexual, segundo coorte de nascimento, para mulheres negras Belo Horizonte - 2002

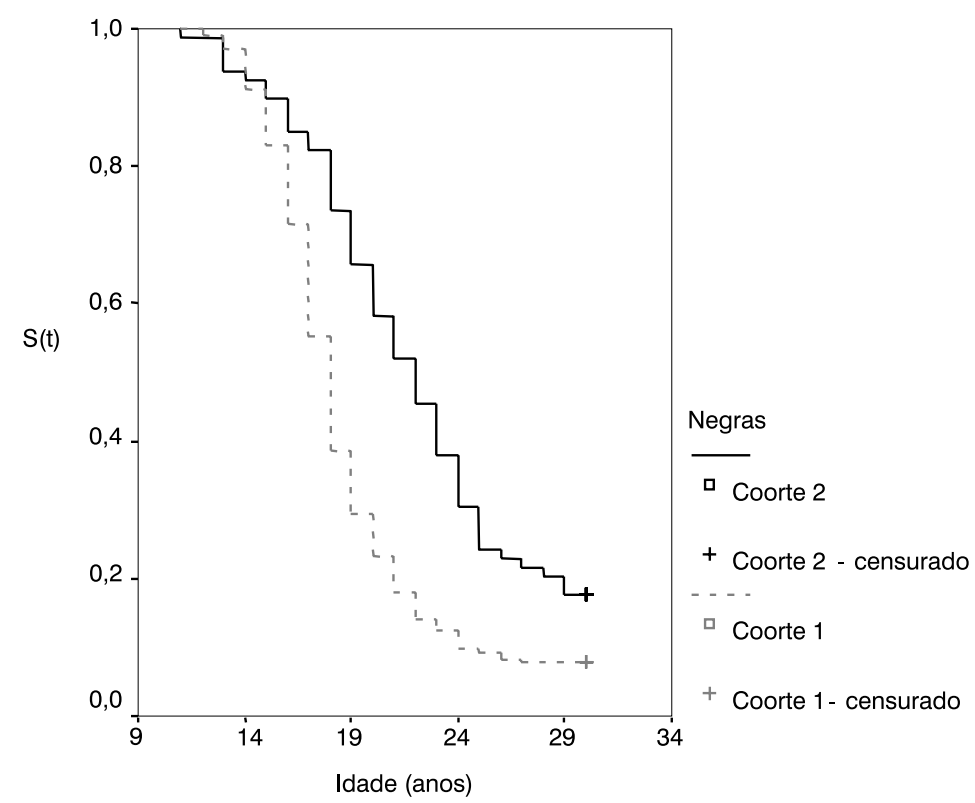

Fonte: Pesquisa SRSR, Cedeplar, Belo Horizonte, 2002. 
A comparação da idade de início da vida sexual das mulheres brancas e negras, da coorte mais jovem, mostra similaridades em relação à idade de início da vida sexual. Os resultados revelam que, tanto para brancas quanto para as negras, a idade mediana de primeira relação sexual é de 18 anos. $O$ teste log-rank mostra que a diferença na idade de primeira relação sexual entre brancas e negras da coorte mais jovem não é estatisticamente significante (valor- $p=0,2127$ ).

Já na coorte 2, até os 17 anos, as muIheres negras iniciavam a vida sexual mais jovens do que as brancas. Para as idades acima dos 17 anos, as brancas tinham a primeira relação sexual mais cedo do que as negras. Entre as mulheres mais velhas, a idade mediana de início da vida sexual para as brancas era de 19 anos e, para as negras, de 22 anos. $O$ resultado do teste log-rank revela que não há diferença estatisticamente significativa entre brancas e negras (valor- $p=0,1944$ ).

A comparação do comportamento da idade de primeira relação sexual de mulheres brancas da coorte 1 e da coorte 2 mostra que as mais jovens iniciaram a vida sexual mais cedo do que as mais velhas, sendo que a idade mediana à primeira relação sexual para as brancas da coorte 1 é de 18 anos e, para as da coorte 2 , corresponde a 19 anos.

O Gráfico 2 permite verificar que, também entre as negras, as mais jovens iniciaram a vida sexual antes das negras da coorte mais velha.

É importante salientar que o resultado do teste log-rank indica que a diferença nestas curvas é significante (valor- $p<0,0001$ ). Quanto à idade mediana de primeira relação, para as negras da coorte 1 é de 18 anos e, para as da coorte 2, 22 anos.

\section{Idade ao primeiro casamento}

Para a interpretação dos resultados relativos à idade à primeira união, é importante lembrar que são incluídas tanto as uniões formais quanto as informais. Neste contexto, a idade mediana de primeira união das mulheres da coorte 1 e da coorte 2 são muito próximas: 22,8 e 23,3 anos, respectivamente. O teste de log-rank confirma que não há diferença nos resultados obtidos para estas duas coortes (valor- $p=0.8055$ ). Entre as mulheres da coorte mais velha, entretanto, as brancas se casavam, pela primeira vez, um ano mais cedo do que as negras, sendo a idade mediana de primeiro casamento das brancas de 22,6 anos e, das negras, de 23,7 anos. A diferença entre a idade de casamento destas duas coortes não é estatisticamente significativa (valor$\mathrm{p}=0,4583$ ). É importante ressaltar que, entre as mulheres mais velhas, a idade ao primeiro casamento era bastante próxima à idade à primeira relação sexual, o que sugere que, diferente das jovens, a iniciação sexual das mulheres da coorte mais velha acontecia no casamento (MIRANDARIBEIRO, 1997).

Entre as mulheres brancas, as idades de entrada na primeira união são praticamente as mesmas: ${ }^{3} 22,3$ anos para a coorte mais jovem e 22,6 anos para a mais velha. O teste log-rank também não foi significativo para este caso (valor- $p=0,9225$ ).

$A$ análise da idade de primeiro casamento para as mulheres negras de ambas as coortes indica que as mais jovens apresentam comportamento muito similar ao das mais velhas. Segundo os dados, as negras da coorte 1 têm uma idade mediana de primeiro casamento de 23,2 anos e as da coorte 2, de 23,7 anos. Também neste caso, a diferença entre a idade de casamento das mulheres destas coortes não é estatisticamente significativa (valor- $p=0,6708$ ).

\section{Idade ao nascimento do primeiro filho}

Em relação à idade ao nascimento do primeiro filho, os resultados indicam que a diferença entre as mulheres da coorte 1 e as da coorte 2 não é significativa (valor$p=0,2284$ ). Vale lembrar, entretanto, que as mulheres mais jovens começaram a vida

\footnotetext{
${ }^{3} \mathrm{~A}$ diferença entre a idade de casamento entre as mulheres destas coortes não é estatisticamente significativa $(v a l o r-p=0,4583)$.
} 
GRÁFICO 3

Curvas de sobrevivência da idade ao nascimento do primeiro filho, segundo coorte de nascimento, para mulheres negras Belo Horizonte - 2002

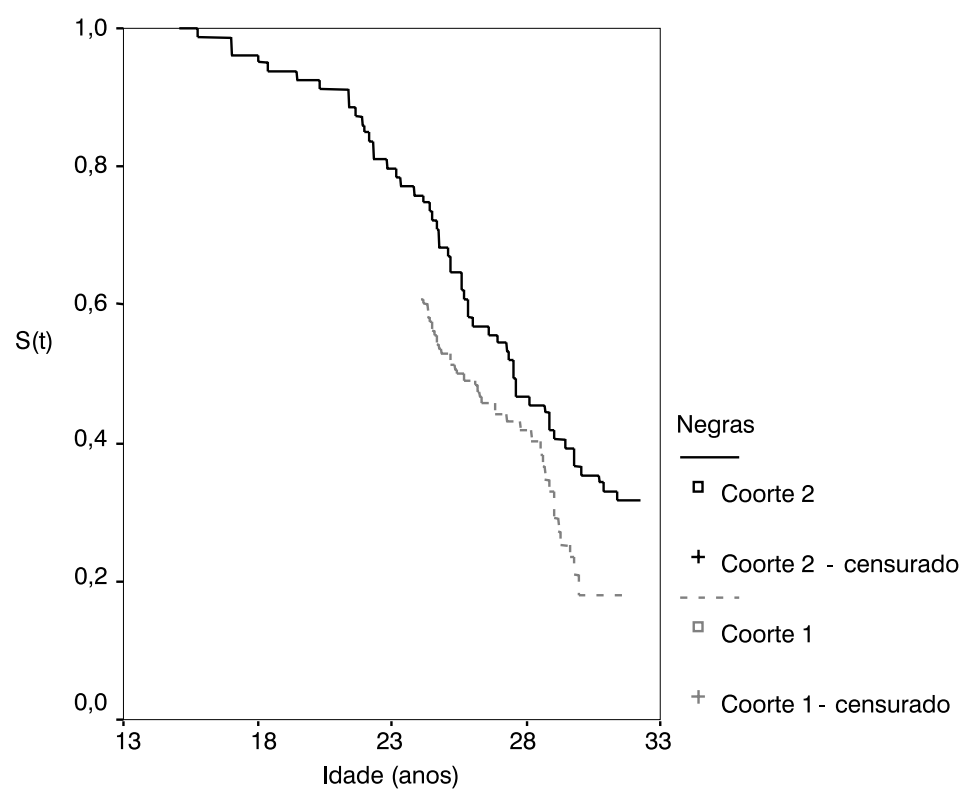

Fonte: Pesquisa SRSR, Cedeplar, Belo Horizonte, 2002.

sexual mais cedo do que as da coorte 2 , sendo plausível que apresentem uma idade de primeiro filho menor, pois iniciaram seu tempo de exposição ao risco de conceber mais cedo do que as mulheres da geração mais velha. A idade mediana de nascimento do primeiro filho é de 24 anos, para a coorte 1, e 24,7 anos, para a coorte 2.

Já os resultados obtidos para a idade ao nascimento do primeiro filho para as brancas e negras da coorte 1 mostram que, embora os dois contingentes femininos apresentem velocidade de início da maternidade muito similar, ela é ainda mais rápida para as negras. A idade mediana corresponde a 25,2 anos, para as brancas, e a 23,2 anos, para as negras. Adicionalmente, é importante esclarecer que o teste logrank, embora não significativo, apresenta probabilidade de significância próxima ao valor limite (valor- $p=0,0730$ ).

A comparação entre as mulheres brancas e negras da coorte 2 revela que, nas idades mais jovens, a probabilidade de as negras terem o primeiro filho antes das brancas é mais elevada. Porém, a partir de cerca de 20 anos, esta probabilidade passa a ser mais elevada para as brancas. $O$ teste log-rank também não foi significativo para este caso $(p=0,3374)$. A idade mediana ao primeiro filho é de 24 anos, para as brancas, e de 25,3 anos, para as negras.

Os resultados alcançados para as muIheres brancas de ambas as coortes indicam que as mais jovens apresentam maior probabilidade de terem o nascimento do primeiro filho mais cedo, quando comparadas às da coorte mais velha, embora a diferença não seja significativa (valor- $p=0,5720$ ). Vale lembrar que a idade mediana de nascimento do primeiro filho é de 25,2 anos para as brancas da coorte 1 e de 24 anos para as da coorte 2.

As curvas relacionadas à idade de nascimento do primeiro filho das mulheres negras sugerem que há diferença significativa entre as duas coortes (valor- $p=0,026$ ). As negras da coorte 1, conforme o Gráfico 3, 
têm probabilidade de terem o primeiro filho mais jovens. Este resultado é compatível com aquele observado para a idade de primeira relação sexual, que indica que as negras mais jovens também iniciaram a atividade sexual mais cedo do que as mais velhas. A idade mediana de nascimento do primeiro filho das negras da coorte 1 é de 23,2 anos, mais baixa que a das negras da coorte 2, de 25,3 anos.

\section{Considerações finais}

Existem diferenças nas idades à primeira relação sexual, à primeira união e ao nascimento do primeiro filho quando se comparam mulheres brancas e negras de diferentes coortes de idade, em Belo Horizonte?

Os resultados sugerem que sim. As mulheres da geração mais jovem estão começando a vida sexual mais cedo do que iniciavam as mais velhas. A diferença na idade de iniciação fica em torno de três anos, sendo 18 anos a idade mediana de primeira relação sexual, para as mais jovens, e 21 anos, para as mais velhas. Uma possível explicação para esta mudança pode estar aliada às transformações sociais e culturais ocorridas no país nas últimas décadas, mencionadas anteriormente, as quais podem ter tornado as normas e valores relativos ao comportamento sexual mais liberais em comparação a tempos passados, permitindo, por exemplo, que as jovens iniciem sua vida sexual antes do casamento.

$\mathrm{O}$ fato de as mulheres mais jovens iniciarem sua vida sexual mais cedo pode ter implicações tanto no plano individual como no social. No plano individual, jovens que iniciam sua vida sexual sem estarem suficientemente esclarecidas a respeito, por exemplo, do papel dos métodos contraceptivos poderão ter que enfrentar desafios, como a ocorrência de infecções sexualmente transmissíveis e uma gravidez não planejada. No plano social, o início da vida sexual por mulheres cada vez mais jovens tem implicações em diversas esferas, entre as quais podem ser citadas a da saúde e a da escola. No âmbito da saúde, os serviços devem estar mais preparados para atender a uma clientela com características e demandas mais específicas. Adicionalmente, as escolas devem repensar a sua postura diante do novo comportamento das jovens, assumindo, em conjunto com a família, um papel mais ativo no que se refere à educação afetivo-sexual, a qual, para acompanhar o rejuvenescimento da idade de iniciação sexual, precisa começar em idades cada vez mais jovens.

Além da idade de início da vida sexual, também constataram-se diferenças no comportamento da idade de nascimento do primeiro filho. Embora as mulheres da geração mais jovem estejam iniciando a vida sexual mais cedo do que as da geração mais velha, elas estão tendo o primeiro filho um pouco mais tarde. Duas hipóteses podem ser cogitadas para explicar este resultado. Primeiro, a maternidade pode estar acontecendo mais tarde, mesmo em vista do início da vida sexual mais cedo, em função do maior acesso à contracepção nos dias atuais. Em segundo lugar, conforme sugerido para o caso do Rio de Janeiro, a maior escolaridade das mulheres mais jovens, em relação às mais velhas, pode funcionar como fonte de conhecimento e de transformação de atitudes em relação ao comportamento sexual, conjugal e reprodutivo. Assim, mulheres mais escolarizadas tendem a adiar a entrada na maternidade.

Os resultados não indicam, porém, diferenças na idade à primeira união das muIheres das diferentes gerações. Ao longo destes 20 a 30 anos que separam estas coortes, a idade de primeiro casamento permaneceu praticamente a mesma, em torno dos 23 anos. Em face das mudanças verificadas nas outras duas idades, as razões da estabilidade na idade de primeiro casamento mereceriam uma investigação mais detalhada.

Quanto às diferenças entre as duas coortes e entre as duas categorias de raça/ cor, os resultados evidenciam que os maiores diferenciais ocorrem entre as duas coortes analisadas. Embora pequenas diferenças na idade de primeiro casamento $e$ de nascimento do primeiro filho ainda persistam entre brancas e negras na geração 
mais jovem, elas diminuíram consideravelmente em relação às diferenças que existiam entre as mulheres da coorte mais velha. Infelizmente, os dados referentes à escolaridade não são retrospectivos e, por isso, não se pode investigar a relação desta variável com os eventos analisados, o que poderia tornar a análise mais rica e informativa. Da mesma forma que Cavenaghi e Alves (2004) afirmam que a escolaridade é mais importante do que a cor da pele na determinação da fecundidade no Rio de Janeiro, os resultados apresentados aqui sugerem que a coorte de nascimento é mais importante que a cor da pele quando se analisam os diferenciais nas idades à primeira relação sexual e ao nascimento do primeiro filho entre as mulheres em Belo

\section{Referências bibliográficas}

ALLISON, P. D. Survival analysis using the SAS system: a practical guide. North Caroline: SAS, 1995.

BEMFAM. Pesquisa nacional sobre demografia e saúde. Rio de Janeiro: Bemfam, 1996.

Pesquisa sobre saúde reprodutiva e sexualidade do jovem: Rio de Janeiro, Curitiba e Recife, 1989/1990. Rio de Janeiro: Bemfam, 1992.

BERCOVICH, A. Considerações sobre a fecundidade da população negra no Brasil. In: LOVELL, P. A. Desigualdade racial no Brasil contemporâneo. Belo Horizonte: MSG, 1991, p. 309-340.

BONGAARTS, J.; POTTER, R. G. Fertility, biology, and behavior: an analysis of the proximate determinants. London: Academy, 1983.

BUMPASS, L. L.; RINDFUSS, R. R.; JANOSIK, R. B. Age and marital status at first birth and the pace of subsequent fertility. Demography, v.15, n.1, p.75-85, 1978.

CARD; J. J. Long-term consequences for children of teenage parents. Demography, v.18, n.2, p.137-156, May 1981.
Horizonte. Isto, no entanto, não quer dizer que o recorte por raça/cor deva ser ignorado em estudos desta natureza. Adicionalmente, cabe pensar que, em face dos resultados encontrados, o efeito coorte pode ser considerado um representante para as possíveis mudanças ocorridas nas normas, valores e acesso à informação e aos serviços quando se comparam as idades de acontecimento dos eventos incluídos na análise.

Por fim, cabe ressaltar que os aspectos aqui investigados apontam fortemente para a importância da realização de um estudo de natureza qualitativa, que pode esclarecer muitas questões relacionadas aos comportamentos sexual, conjugal e reprodutivo das mulheres em Belo Horizonte.

CARVALHO, J. A.; WOOD, C.; ANDRADE, F. D. Notas acerca das categorias de cor dos censos e sobre a classificação subjetiva de cor no Brasil: 1980/90. ENCONTRO NACIONAL DE ESTUDOS POPULACIONAIS, 12, Caxambu, 2000. Anais... Disponível em:<http://www.abep.org.br/usuario/ GerenciaNavegacao.php?caderno_id= 188\&nivel=2>. Acesso em: 16 abr. 2002.

CAVENAGHI, S. M.; ALVES, J. E. D. A dinâmica da fecundidade no Rio de Janeiro: 1991-2000. Trabalho apresentado no Seminário Tendências da Fecundidade e Direitos Reprodutivos no Brasil, 1, 2004, Belo Horizonte.

DAVIS, K.; BLAKE, J. Social structure and fertility: an analytic framework. Economic Development and Cultural Change, v.4, n.3, p.211-235, Apr. 1956.

FARIA, V. E. Políticas de governo e regulação da fecundidade: conseqüências não antecipadas e efeitos perversos. Ciências Sociais Hoje, 1989: anuário de antropologia, política e sociologia. São Paulo: Vértice/Anpocs, 1989, p. 62-103.

FARIA, V. E.; POTTER, J. E. Development, government policy, and fertility regulation 
in Brazil. Austin: The University of Texas at Austin, 1990 (Paper, 12.02).

FRIAS, L. A. de M.; CARVALHO, J. A. M. de. Fecundidade nas regiões brasileiras a partir de 1903: uma tentativa de reconstrução do passado. ENCONTRO NACIONAL DE ESTUDOS POPULACIONAIS, 9, Caxambu, 1994. Anais... Belo Horizonte: ABEP, v.2, 1994, p.23-46.

GUPTA, N., LEITE, I. da C. Tendências e determinantes da fecundidade entre adolescentes no Nordeste do Brasil. Perspectivas Internacionais de Planejamento Familiar, volume especial, p.24-29, 2001.

HOPES and Realities. Closing the gap between women's aspirations and their reproductive experiences. New York: The Alan Guttmacher Institute, 1995.

MARTIN, T. C., JUÁREZ, F. The impact of women's education on fertility in Latin America: Searching for explanations. International Family Planning Perspectives, v.21, n.2, p.52-57, Jun. 1995.

MERRICK, T. W., BERQUÓ, E. The determinats of Brazil's recent rapid decline in fertility. Washington: National Academy, 1983.

MIRANDA-RIBEIRO, P. Telenovelas and the sexuality transition among teenagers in Brazil. 1997. Tese de doutorado em sociologia - The University of Texas, Austin, 1997.

MIRANDA-RIBEIRO, P.; CAETANO, A. O programa SRSR. Revista Brasileira de Estudos Populacionais, v.20 n.2, p.303-305, jul./dez. 2003. Disponível em: <http:// www.abep.nepo.unicamp.br/docs/rev_inf/ vol20_n22003_11notapesquisa_p303a305.pdf > Acesso em: set. 2004.

MOORE, A. M. Female control over first sexual intercourse in Brazil: case studies of Belo Horizonte, Minas Gerais and Recife, Pernambuco. 2004. Tese de doutorado em filosofia - The University of Texas at Austin, Austin, 2004.
OLIVEIRA, F. O recorte racial/étnico e a saúde reprodutiva: mulheres negras. In: GIFFIN, K.; COSTA, S. H (Orgs.). Questões da saúde reprodutiva. Rio de Janeiro: FIOCRUZ, 1999, p. 419-438.

PERPÉTUO, I. H. O. Raça e acesso às ações prioritárias na agenda da saúde reprodutiva. ENCONTRO NACIONAL DE ESTUDOS POPULACIONAIS, 12, Caxambu, 2000. Anais... Disponível em: <http://www.abep. org.br/usuario/GerenciaNavegacao.php? caderno_id $=188 \&$ nivel $=2>$. Acesso em ago. $200 \overline{4}$.

POPULATION handbook. Washington: Population Reference Bureau, 1992.

RELATÓRIO SRSR. Belo Horizonte: UFMG/ Cedeplar, 2003. Mimeografado.

SIMÃO, A. A primeira relação sexual, o primeiro casamento e o nascimento do primeiro filho: um estudo quantitativo e qualitativo de duas coortes de mulheres em Belo Horizonte. 2005. Tese de doutorado em demografia-Cedeplar: Belo Horizonte, 2005.

SIMÕES, C. C. da S.; OLIVEIRA, L. A. P. Perfil estatístico de crianças e mães no Brasil: a situação da fecundidade; determinantes gerais e características da transição recente. Rio de Janeiro: IBGE, 1988.

TEACHMAN, J.; HECKERT, D. A. The declinng significance of first birth timing. Demography, v.22, n.2, p.185-198, 1985.

TELLES, E. Racismo à brasileira: uma nova perspectiva sociológica. Rio de Janeiro: Relume-Dumará - Fundação Ford, 2003.

WONG, L. L. A projeção da fecundidade um exercício aplicado para o Brasil para o período 1991-2020. ENCONTRO NACIONAL DE ESTUDOS POPULACIONAIS, 12, Caxambu, 2000. Anais... Disponível em: <http://www.abep.org.br/usuario/Gerencia Navegaçao.php?caderno_id188\&nivel $=2>$ Acesso em: 21 jul.2003. 


\begin{abstract}
Comparing the Ages at First Intercourse, First Union and First Childbirth of Two Cohorts of White and Mixed-race Females in Belo Horizonte, Brazil: Quantitative Indications
\end{abstract}

This paper investigates the ages at first sexual intercourse, first marriage and first childbirth of white and mixed-race (black + brown) females, in the age groups of 20 to 29 and 50 to 59 , in the city of Belo Horizonte, Brazil, in 2002. The objectives are twofold. The first aim is to verify the changes in the age at first sexual intercourse, first marriage and first childbirth. The second is to investigate the role of birth cohort and ethnic group /skin color in the differences seen. The data was taken from the survey entitled "Reproductive Health, Sexuality and Color/Ethnic Group [Saúde Reprodutiva, Sexualidade e Raça/Cor]" (SRSR), conducted by Cedeplar in 2002. The methodology is based on survival curves, obtained by Kaplan-Meier estimates, and compared by log-rank tests.

Key Words: First sexual intercourse. First marriage. First childbirth. Age. Ethnic group and skin color. Belo Horizonte, Brazil.

Recebido para publicação em 03/05/2005.

Aceito para publicação em 14/10/2005. 